\title{
Overexpression of DICER1 induced by the upregulation of GATA1 contributes to the proliferation and apoptosis of leukemia cells
}

\author{
YING BAI $^{1,2}$, GUANG-RONG QIU ${ }^{1}$, FAN ZHOU ${ }^{2}$, LI-YING GONG ${ }^{1,3}$, FENG GAO $^{1,4}$ and KAI-LAI SUN ${ }^{1}$ \\ ${ }^{1}$ Department of Medical Genetics, China Medical University; ${ }^{2}$ Department of Hematology, \\ Shenyang General Military Hospital; ${ }^{3}$ Department of Laboratory Medicine, Liaoning Provincial People's Hospital; \\ ${ }^{4}$ Department of Hematology, First Affiliated Hospital of China Medical University, Shenyang, P.R. China
}

Received November 19, 2012; Accepted January 17, 2013

DOI: 10.3892/ijo.2013.1831

\begin{abstract}
Dicer, a member of the RNase III family, is the key enzyme required for the biogenesis of microRNAs and small interfering RNAs. Recent evidence indicates that DICERI expression levels vary among different solid tumors and decreased or increased DICERI expression has been associated with aggressive cancers. In this study, we assessed DICERI expression levels in acute myeloid leukemia (AML) and investigated its biological effects and transcriptional regulation in leukemia cell lines. We demonstrated that DICERI was overexpressed in AML patients and leukemia cell lines by realtime quantitative PCR and western blot analysis. A functional assay demonstrated that the silencing of DICERI inhibited cell proliferation and promoted apoptosis in leukemia cell lines. We also demonstrated that DICERI was upregulated by the hematopoietic transcription factor, GATA1, through luciferase, electrophoretic mobility shift and chromatin immunoprecipitation assays. These data suggest that DICER1 plays an important role in AML and the finding that the upregulation of DICERI is induced by GATA1 may provide a framework for the understanding of differential DICERI expression levels in multiple types of cancer.
\end{abstract}

\section{Introduction}

Dicer, a highly conserved RNase III-type enzyme found in the majority of eukaryotes (1), cleaves microRNA (miRNA) and small interfering RNA (siRNA) precursors into approximately 22 nucleotides $(2,3)$. It is the key enzyme for RNAi and miRNA pathways. Contrary to other organisms, mammals have a single species of Dicer, which is involved in processing precursor double-stranded RNA (dsRNA) into miRNA and siRNA (4).

Correspondence to: Dr Kai-Lai Sun, Department of Medical Genetics, China Medical University, Shenyang, P.R. China

E-mail: klsun@vip.sina.com

Dr Ying Bai, Department of Hematology, Shenyang General Military Hospital, Shenyang, P.R. China

E-mail: baiying3939@126.com

Key words: DICER1, acute myeloid leukemia, GATA1, regulation
Over the past several years, Dicer has increasingly evoked interest in the field of cancer biology, based on a number of studies demonstrating the aberrant expression of DICERI among different types of cancer (5-8). Furthermore, the abnormal expression of DICER1 has been associated with poor prognosis in cancer patients (9-12). Despite growing evidence that Dicer plays a significant role in tumorigenesis, the regulation of the expression of this gene remains to be elucidated. As regards hematological malignancies, the potential role of Dicer and the regulation of its expression have not been widely investigated. Therefore, in this study, we assessed DICERI expression levels in acute myeloid leukemia (AML) cells and investigated whether alterations in DICERI expression play an important role in AML cell proliferation and apoptosis. In addition, we investigated the mechanism behind the dysregulation of DICER1 expression in AML cells.

\section{Materials and methods}

Patients, samples and reagents. A total of 42 untreated de novo AML patients (non-M3) with $\geq 80 \%$ leukemia cells and 5 healthy volunteers were included in this study. According to the French-American-British (FAB) classification, the patient subtypes were as follows: 1 patient had M0, 2 had M1, 5 had M2, 9 had M4, 22 had M5 and 3 had M6. At the time of recruitment, informed consent was obtained from each subject, in accordance with the declaration of the hospital authorities. This study was approved by the Research Ethics Committee of China Medical University, Shenyang, China. Samples were collected from patients at the Department of Hematology of Shenyang General Military Hospital, Shenyang, China. Leukemia bone marrow mononuclear cells (BMMCs) were enriched from the diagnostic bone marrow samples of patients with de novo AML by Ficoll-Hypaque (Sigma-Aldrich, St. Louis, MO, USA) density gradient centrifugation. Purification of $\mathrm{CD} 34^{+}$hematopoietic progenitor cells in normal BMMCs was performed by positive selection using the MACS Direct CD34 Progenitor Cell Isolation kit (Miltenyi Biotec, Bergish Gladbach, Germany) as the control.

Cell culture. The U937 and K562 human leukemia cell lines were suspended in RPMI-1640 (Invitrogen, Carlsbad, CA, USA). Human embryonic kidney 293 (HEK293) cells, were cultured in DMEM (Gibco, Carlsbad, CA, USA). All media 
contained standard antibiotics and $10 \%$ fetal bovine serum (HyClone Laboratories, Logan, UT, USA). The cells were incubated at $37^{\circ} \mathrm{C}$ in a humidified atmosphere of $5 \% \mathrm{CO}_{2}$.

Real-time quantitative PCR. Total RNA was isolated using TRIzol reagent (Invitrogen). cDNA was prepared from 1-2 $\mu \mathrm{g}$ of total RNA using SuperScript II Reverse Transcriptase (Invitrogen). cDNA $(2 \mu \mathrm{l})$ was used for real-time PCR, which was performed to detect DICERI using SYBR-Green (Takara, Shiga, Japan), according to the manufacturer's instructions. Sequences of primers were as follows: DICER1, 5'-GTACGA CTACCACAAGTACTTC-3' and 5'-ATAGTACACCTGCCA GACTGT-3'; GATA1, 5'-CAGTAAACGGGCAGGTACTC-3' and 5'-CATAAAGCCACCAGCTGGTC-3'; and GAPDH, 5'-TGCACCACCAACTGCTTAG-3' and 5'-GACGCAGGG ATGATGTTC-3'. Normalization was performed with respect to GAPDH levels. Real-time PCR consisted of an initial denaturation at $95^{\circ} \mathrm{C}$ for $15 \mathrm{sec}$, followed by 40 cycles at $95^{\circ} \mathrm{C}$ for $5 \mathrm{sec}$ and $60^{\circ} \mathrm{C}$ for $34 \mathrm{sec}$. Each sample was analyzed in triplicate, using ABI 7500 System software. The $2^{-\Delta \Delta \mathrm{Ct}}$ method was used to calculate relative changes in gene expression.

Western blot analysis. Cells were lysed in RIPA buffer and the protein concentration was determined using the Bradford method with BSA (Sigma-Aldrich) as the standard (Bio-Rad, Hercules, CA, USA). Equal amounts of cell extract (50 $\mu \mathrm{g})$ were subjected to electrophoresis on a SDS-polyacrylamide gel and transferred onto a PVDF membrane. The membrane was blocked and then incubated with primary antibodies against DICER1 (Santa Cruz Biotechnology, Santa Cruz, CA, USA), $\alpha$-tubulin, GAPDH (BiYunTian Biotechnology Co., Ltd., Shanghai, China) and GATA1 (Abcam), followed by an HRP-conjugated secondary antibody. The blots were developed using chemiluminescence.

RNAi assays. The K562 and U937 human leukemia cells were transfected with an shRNA expression vector. The DICER1shRNA and control-shRNA vectors were purchased from Shanghai GenePharma, Co., Ltd., Shanghai, China. The DICER1-shRNA sequence used was 5'-GCTCGAAATC TTACGCAAATATTCAAGAGATATTTGCGTAAGATTTC GAGCTT-3'. The GATA1-shRNA sequence used was 5'-CTC AATTCAGCAGCCTATTCTCGAGAATAGGCTGCTGAA TT GAG-3'. Transfection was performed with Lipofectamine ${ }^{\mathrm{TM}}$ LTX and PLUS ${ }^{\mathrm{TM}}$ Reagents (Invitrogen) according to the manufacturer's instructions. The cells were harvested 5 days after transfection and used for further assays.

Cell growth assay. Cell proliferation was assessed using a standard CCK-8 assay (Dojindo, Kunamoto, Japan). Absorbance was determined at a test wavelength of $450 \mathrm{~nm}$ on a multi-well plate reader (Microplate Reader; Bio-Rad, Hercules, CA, USA).

Apoptosis assay. Cell apoptosis was assayed by staining with Annexin V-FITC (KeyGEN, Nanjing, China), according to the manufacturer's instructions and detected by flow cytometry on a FACSCalibur (Becton-Dickinson, Franklin Lakes, NJ, USA).

Plasmid construction. The construction of a series of truncated constructs containing the human DICERI promoter was performed as follows: A 1,062-bp fragment of the human DICER I promoter was generated through PCR, using as a template genomic DNA obtained from human blood samples. The DICERI promoter PCR primers included a forward primer, starting at $-975 \mathrm{bp}$ (where +1 is the transcription start site of DICER 1), a reverse primer, starting at $+55 \mathrm{bp}$ and several smaller forward primers: primer 975, 5'-TCCGGTACC TATCTCGATTCCGACTAGC-3'; primer 652, 5'-TCCGGT ACCCAGCTTACAAACAGAGGGCCA-3'; primer 489, 5'-TCCGGTACCGAGGTGCTCAGAGGGAAGCTAA-3' and primer 208, 5'-TCCGGTACCGATTAACCTTTCACCGCC AGG-3'. The sequence of the reverse primer was 5'-GGCAAG CTTCGCAACCCTCATTAAAAGAGC-3'. The amplified fragments were attached to the KpnI and HindIII digestion sites of the pGL3-basic vector (Promega, Madison, WI, USA). The human GATAl expression vector (pcDNA3.1-GATA1, pGATA1) was purchased from GenScript Corp. - Biology CRO, Piscataway, NJ, USA.

Luciferase assay. Cells in 24-well plates were transfected in triplicate using Lipofectamine LTX and PLUS Reagents (Invitrogen) with $1 \mu \mathrm{g}$ of specific plasmids. After $48 \mathrm{~h}$ of transfection, cells were harvested in $100 \mu \mathrm{l}$ of Passive Lysis buffer (Promega) and the luciferase assay was performed using the Dual Luciferase Assay system (Promega), according to the manufacturer's instructions. Relative luciferase activity was calculated as the ratio of firefly luciferase activity to Renilla luciferase activity.

Electrophoretic mobility shift assay (EMSA). Preparation of nuclear extracts from K562 and U937 cells was performed using an Active Motif Nuclear Extract kit (Active Motif, Carlsbad, CA, USA) according to the manufacturer's instructions. The following double-stranded oligonucleotides were used in the present study (wild-type and mutant binding sites are underlined): Wild-type, 5'-tctcaTTATCTgggga-3'; and mutant, 5'-tctcatGCGTtgggga-3'. Oligonucleotide labeling was performed using the Biotin 3' End Labeling kit (Pierce Biotechnology, Rockford, IL, USA). The LightShift Chemiluminescent EMSA kit (Pierce Biotechnology) was used according to the instructions provided. For competition assays, samples were pre-incubated with a 100 -fold excess of the unlabeled wild-type or mutated oligonucleotide duplex competitors. For the supershift reaction, $1 \mu \mathrm{g}$ of anti-GATA1 antibody (Abcam) was pre-incubated with the nuclear extracts for $30 \mathrm{~min}$ on ice.

Chromatin immunoprecipitation assay (ChIP). ChIP assays were performed as previously described $(13,14)$. Briefly, cells were cross-linked with $1 \%$ formaldehyde for $10 \mathrm{~min}$ at $37^{\circ} \mathrm{C}$. After washing with ice-cold PBS, cells were lysed with lysis buffer containing protease inhibitors and sonicated to obtain DNA fragments. A sample of untreated DNA fragments was saved as the input DNA. The remaining sample was diluted, treated with anti-GATA1 antibody (Abcam) or rabbit IgG and immunoprecipitated by Protein A/G Plus-Agarose (Santa Cruz Biotechnology). Each precipitated sample was eluted and extracted with phenol-chloroform and subjected to PCR. PCR was performed using DICER1 promoter-specific primers amplifying the GATA1-binding regions (forward 

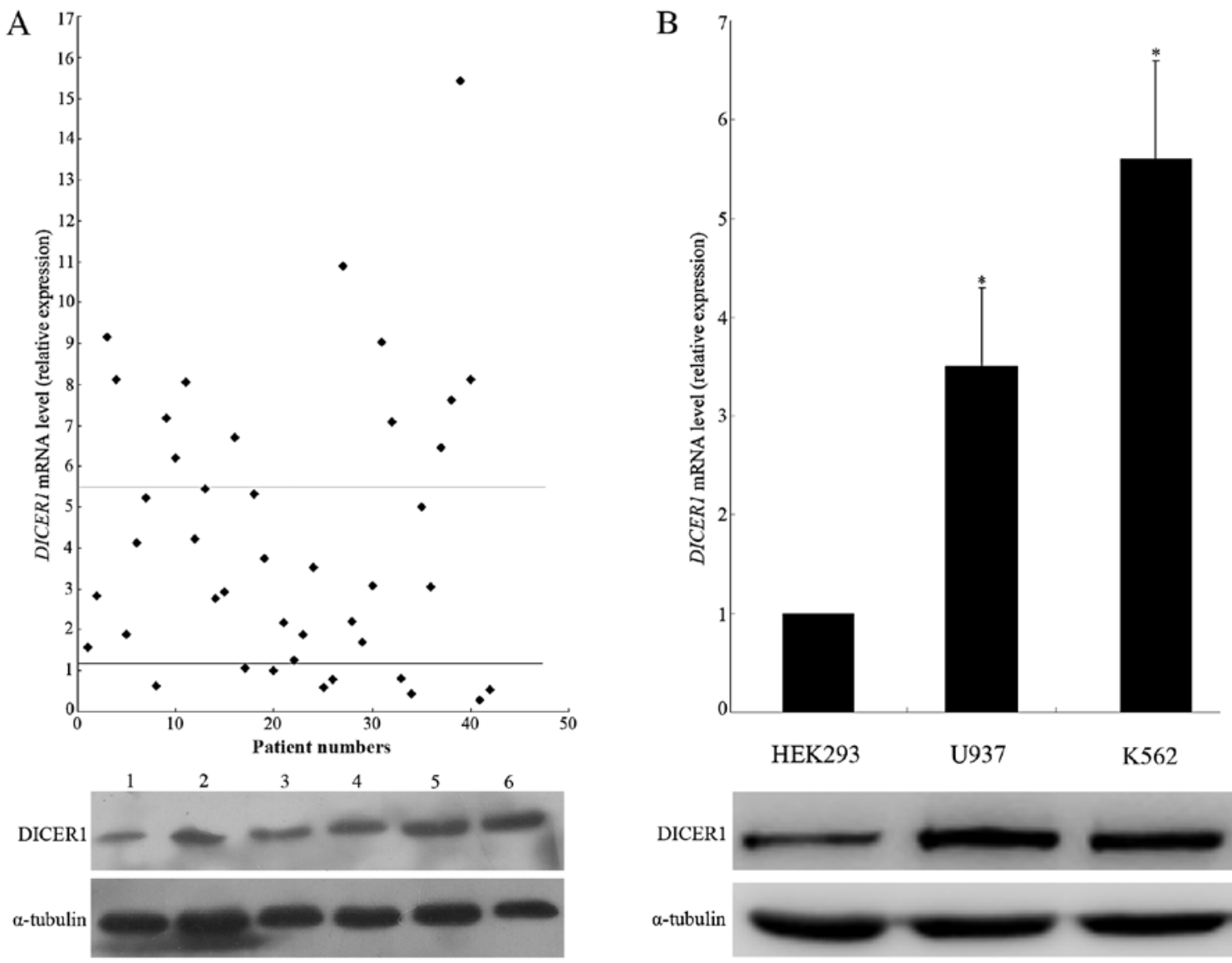

Figure 1.DICER1 expression levels in adult AML blast cells. (A) DICER1 mRNA expression levels. The DICER1 mRNA levels of 42 AML samples were measured by real-time quantitative PCR and normalized to GAPDH expression. The black bar represents mean expression values (1.2 \pm 0.81$)$ of normal controls. The gray bar represents mean increased DICER1 mRNA values (5.49 \pm 3.18 ). The expression of DICER1 mRNA was significantly elevated in AML cases compared to the normal donors $(\mathrm{P}<0.01)$ (top panel). $\mathrm{P}$-values were calculated using the Mann-Whitney $\mathrm{U}$ test. The expression levels of the DICER1 protein in AML patients and normal controls were determined by western blot analysis (bottom panel). Lane 1, normal controls; lanes 2-6, AML cases. (B) Expression of DICER1 mRNA and protein in HEK293 cells and U937 and K562 human leukemia cell lines. ${ }^{*} \mathrm{P}<0.05$.

primer, 5'-CTGGGGATCCCGGTTGG-3'; reverse primer, 5'-GCATTGTTGCTCCTTCTG-3').

Statistical analysis. Data were subjected to statistical analysis and are presented as the means \pm standard error of the mean (SEM). Differences in mean values were analyzed using one-way analysis of variance (ANOVA). For the comparison of DICER1 mRNA levels between AML cases and normal donors, the Mann-Whitney U test was used. All analyses were performed with SPSS Statistics version 18.0 software (SPSS Inc., Chicago, IL, USA). In the figures, P-values indicating a statistically significant difference are marked with asterisks $\left({ }^{*} \mathrm{P}<0.05,{ }^{* *} \mathrm{P}<0.01\right)$.

\section{Results}

High expression of DICERI in AML patients. We examined bone marrow (BM) samples of 42 AML cases and normal donors using real-time quantitative PCR for DICERI mRNA. Compared to the normal controls, we observed an upregulation of DICER1 mRNA expression in the bone marrow of AML patients with statistically significant differences $(\mathrm{P}<0.01$, Fig. 1A). The mean increased DICERI mRNA expression level in AML patients was 4.58-fold higher compared to that in the normal controls and the increased DICERI mRNA level was defined as higher than the mean expression level $(1.2 \pm 0.81)$ of the normal controls. In the 19 cases in which DICERI mRNA expression was increased, the DICERI protein level was detected by western blot analysis. The results showed that the expression of the DICERI protein was markedly elevated in a significant proportion of AML cases (74\%), compared to the normal controls. In addition, we assessed DICER 1 expression in the K562 and U937 human leukemia cell lines, in which we observed a significantly increased expression of DICERI at the mRNA and protein levels, compared to HEK293 cells (Fig. 1B).

Silencing of DICER1 induces growth arrest and promotes apoptosis in leukemia cell lines. To validate the functional role of DICERI in leukemia cells, we used DICERI-shRNA to knock down DICERI in 2 leukemia cell lines (K562 and U937). Real-time quantitative PCR and western blot analysis confirmed DICERl knockdown in both cell lines. As shown in Fig. $2 \mathrm{C}$, cell proliferation experiments revealed that DICERI silencing inhibited the proliferation of K562 and U937 cells. We further investigated the effects of DICER1 silencing on cell apoptosis in both cell lines. To that end, apoptotic cells were assessed by Annexin V-FITC staining, followed by flow cytom- 
A
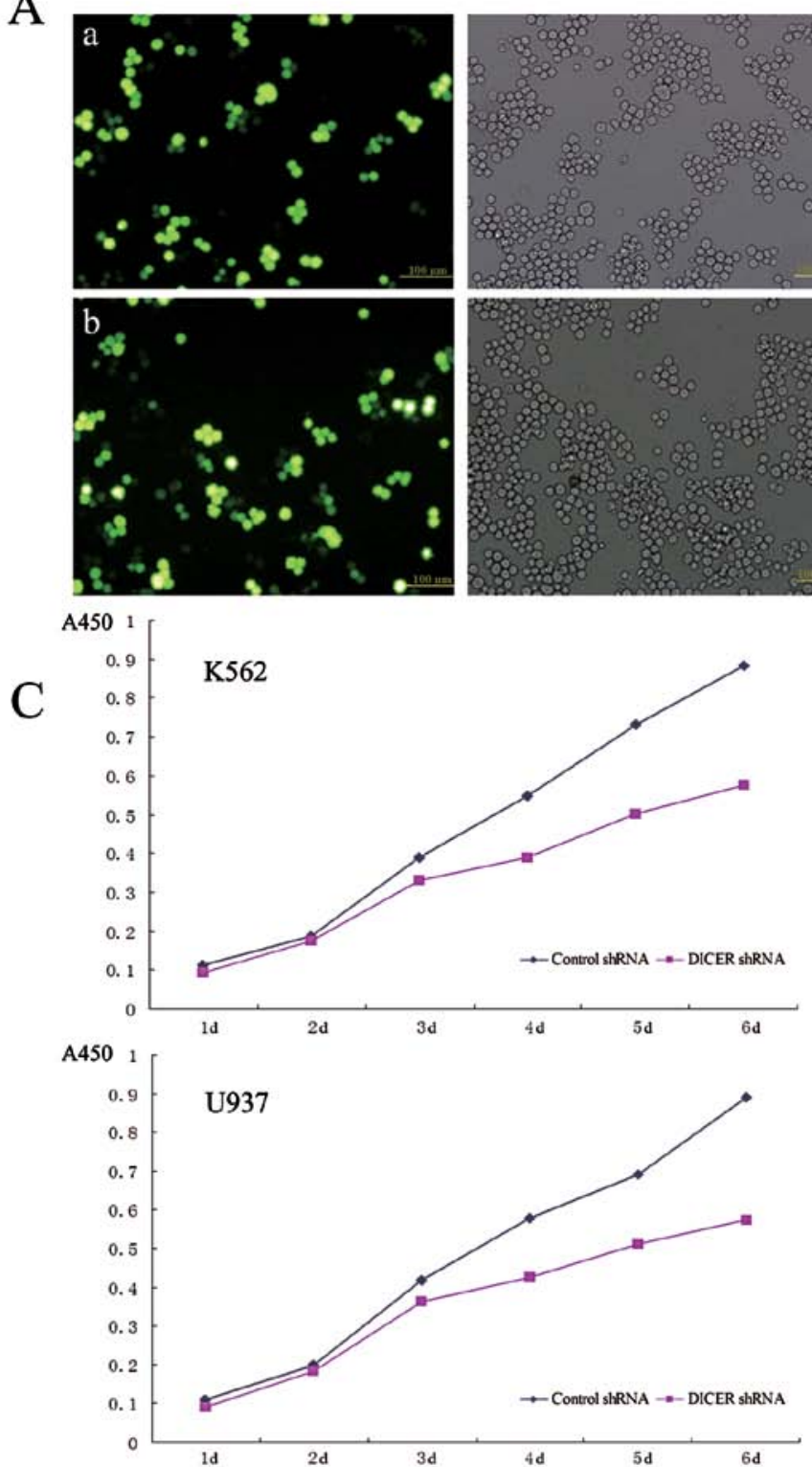

B

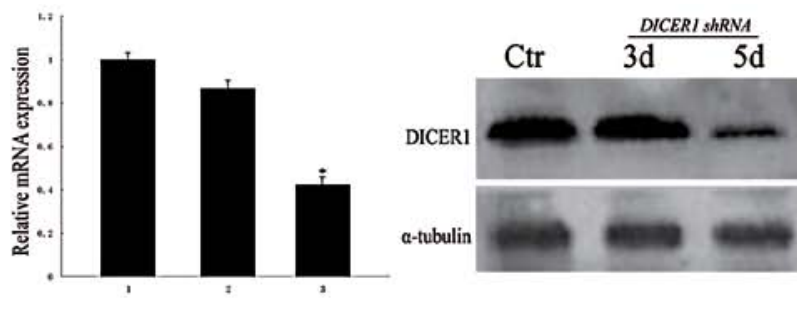

K562

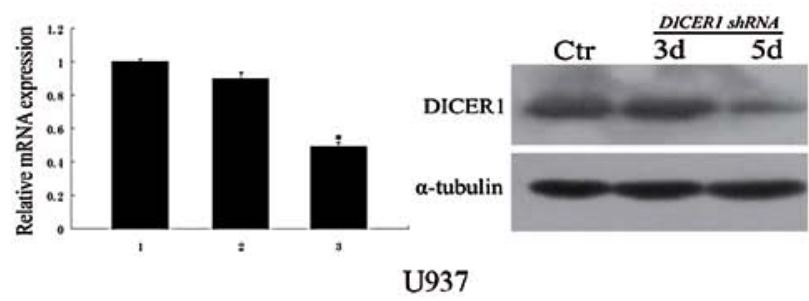

$\mathrm{D}$

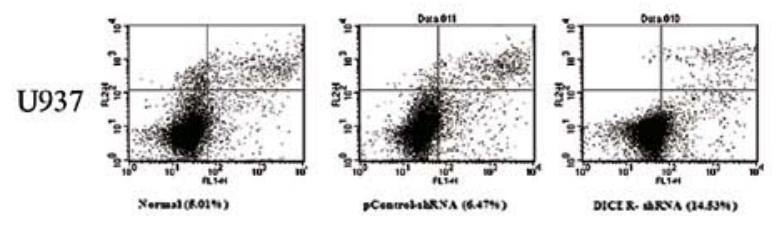

K562
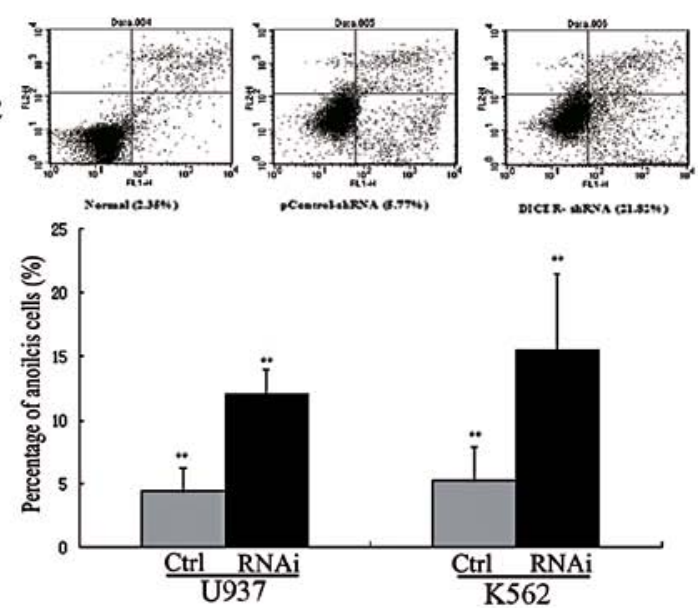

Figure 2. Effect of DICER1 silencing on leukemia cell growth and apoptosis. (A) The K562 and U937 leukemia cells transfected with DICER1-shRNA for $24 \mathrm{~h}$ were examined by fluorescence interference-contrast microscopy at the same phase of the cell cycle (a) K562 cells (b) U937 cells. (B) Knockdown of DICERI in the K562 and U937 leukemia cell lines. Compared to control-shRNA, the levels of DICER1 mRNA and protein were significantly decreased in both cell lines 5 days following transfection with DICER1-shRNA (Ctr, control group; $3 \mathrm{~d}, 3$ days after transfection with DICER1-shRNA; 5d, 5 days after transfection with DICER1-shRNA). "P<0.05. (C) Effect of downregulation of DICER1 on leukemia cell growth. K562 and U937 cells transfected with DICER1-shRNA and control-shRNA were seeded in 96-well plates and cell growth was assayed daily for 6 days. (D) K562 and U937 cell apoptosis following inhibition of DICERI expression by RNAi." $\mathrm{P}<0.01$.

etry. Flow cytometry analysis demonstrated that the silencing of DICER1 significantly induced apoptosis compared to the controls $(\mathrm{P}<0.01$, Fig. 2D). The percentages of early apoptotic U937 cells in the blank, control-shRNA and DICER-shRNA groups were $5.01 \pm 1.25,6.47 \pm 2.06$ and $14.53 \pm 1.81 \%$, respectively; similar results were observed in the K562 cells: $2.35 \pm 0.84,5.77 \pm 1.62$ and $21.82 \pm 2.36 \%$, respectively.

Analysis of the regulation of DICERI expression as well as that of its promoter. Previous studies have demonstrated that specific types of tumors exhibit differential expression of DICERI (5-8), suggesting the tissue-specific regulation of its expression. In this study, we demonstrated that DICERI expression in AML patients was increased and that DICERI mRNA expression correlated with its protein expression. Therefore, we hypothesized that in AML patients, the upregulation of DICER1 may be associated with hematopoietic-specific regulation. The regulation of DICERI expression appeared to occur mainly at the transcriptional level. We analyzed $1,300 \mathrm{bp}(-1200$ to +100$)$ of the 5'-flanking region sequence of DICERI using TRANSFACtranscription element search software (TESS) and discovered 1 GATA1-binding element (-617 to -611) in this region (Fig. 3). GATA1 is a zinc-finger transcription factor that plays an important role in gene regulation during the development and differentiation of hematopoietic cells. The tissue-specific expression of GATA1 is mainly restricted to hematopoietic and 


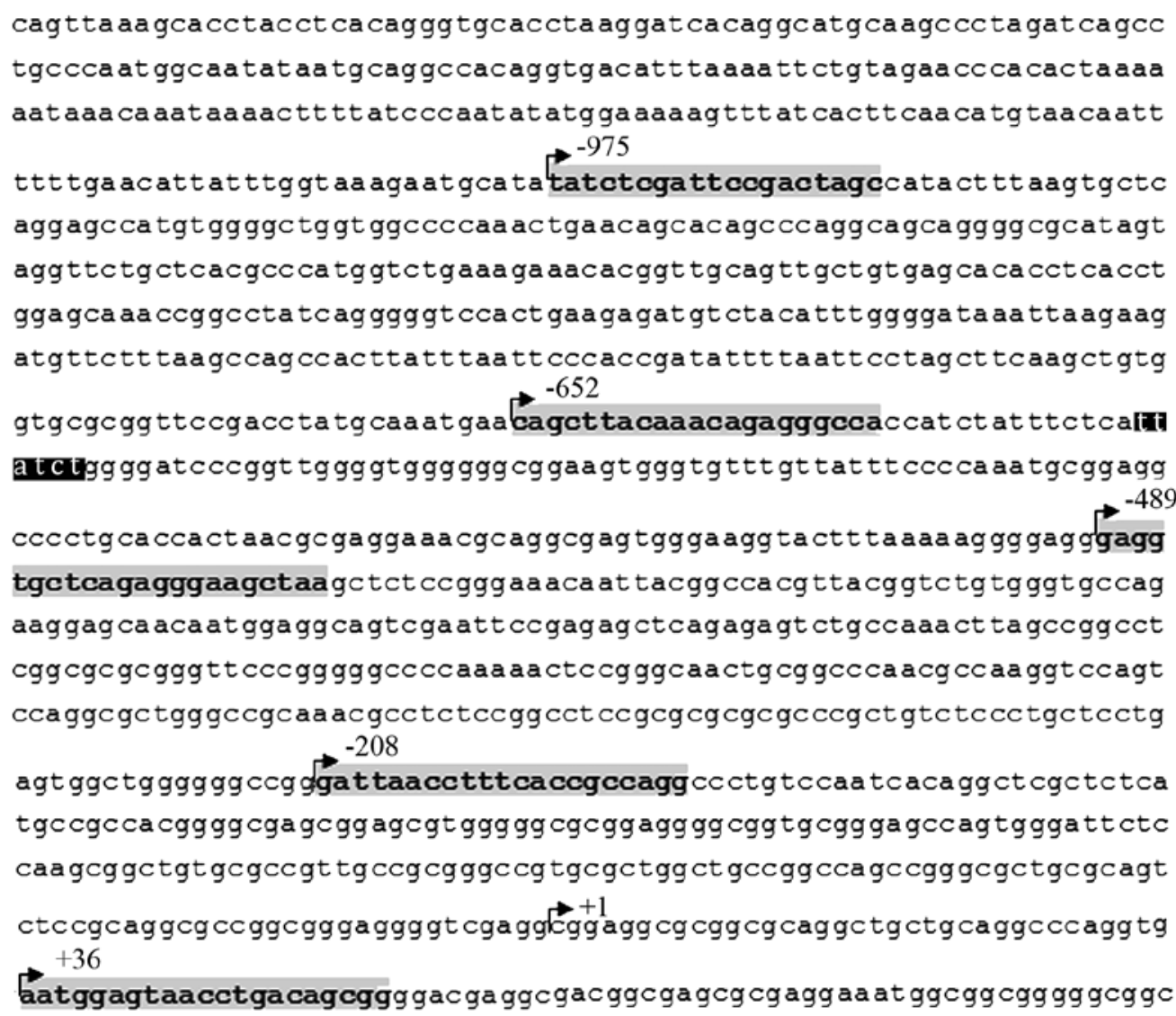

Figure 3. Sequence of the 5'-flanking region of the human DICER1 promoter. Gray areas denote the primers used to generate a series of truncated constructs. The putative GATA1 binding site is shown in black. +1 , transcriptional start site.

Sertoli cells $(15,16)$. Previous studies have demonstrated that leukocytes from AML patients express a significant amount of GATA1 (17-19) and that the overexpression of GATA1 exerts a negative effect on the event-free survival of AML patients $(20,21)$. These data led us to explore the possibility that GATA1 may directly upregulate DICERI in AML.

GATAl binds to the DICER1 promoter. In order to determine whether GATA1 binds to the DICERI promoter, we performed EMSAs using nuclear protein from K562 and U937 cells and the oligonucleotide probe containing the GATA1 sites. As shown in Fig. 4A, nuclear protein from K562 and U937 cells bound to the labeled wild-type oligonucleotide probe and binding was diminished with the addition of 100X unlabeled wild-type and labeled mutant oligonucleotide probes. Furthermore, the bands were supershifted by specific GATA1 antibodies. The interaction between GATA1 and the DICERI promoter was further confirmed by ChIP assay. Primers were used to amplify the GATA1-binding regions of the DICERI promoter. We observed PCR products when using the GATA1 antibody-precipitated samples from the K562 and U937 cells, but not in the negative control immunoprecipitation samples when using the anti-rabbit IgG antibody (Fig. 4B).

GATA1 transactivates the DICER1 promoter. EMSA and ChIP studies confirmed that GATA1 bound to the DICERI promoter; therefore, we sought to determine whether GATA1 can transactivate the DICER1 promoter. We cloned the human
DICER1 promoter (1,062 bp) into a luciferase reporter plasmid (pGL3-Basic) and generated a series of truncated constructs (Fig. 5A, left panel). The locations of the primers of these constructs are shown in Fig. 3 (gray areas). The generated fragments were transiently co-transfected into the K562 and U937 cells in conjunction with pRL-TK. Luciferase assay results revealed an approximately 2 -fold increase in the luciferase activity of p652 compared to that of p484, indicating that the region spanning between -652 and -484 bp contained one or more positive regulatory elements. The GATA1-binding sites (-617 to -611) are located in this region; therefore, to confirm that the GATA1 sites contributed to DICERl transcription, we co-transfected a GATAl expression vector (pcDNA3.1-GATA1, pGATA1) and a p652 vector into the K562 and U937 cells. The relative luciferase activity in the p652 + pGATA1 group was significantly increased compared to that in the p652 + empty pcDNA3.1 (pCtr) group on the 3rd day post-transfection $(\mathrm{P}<0.01$, Fig. 5B). These results indicate that GATA1 positively regulates DICERI promoter expression.

GATAl upregulates DICERl expression in AML. Since our luciferase reporter, EMSA and ChIP assays described above indicated that GATA1 upregulated DICERI transcription, we attempted to verify that GATA1 regulated DICERI expression. To that end, K562 and U937 cells were transfected with a GATA1 expression construct for 2 days. The forced expression of GATA1 increased DICER1 expression at 2 days post-transfection $(\mathrm{P}<0.05$, Fig. 6A). Furthermore, 


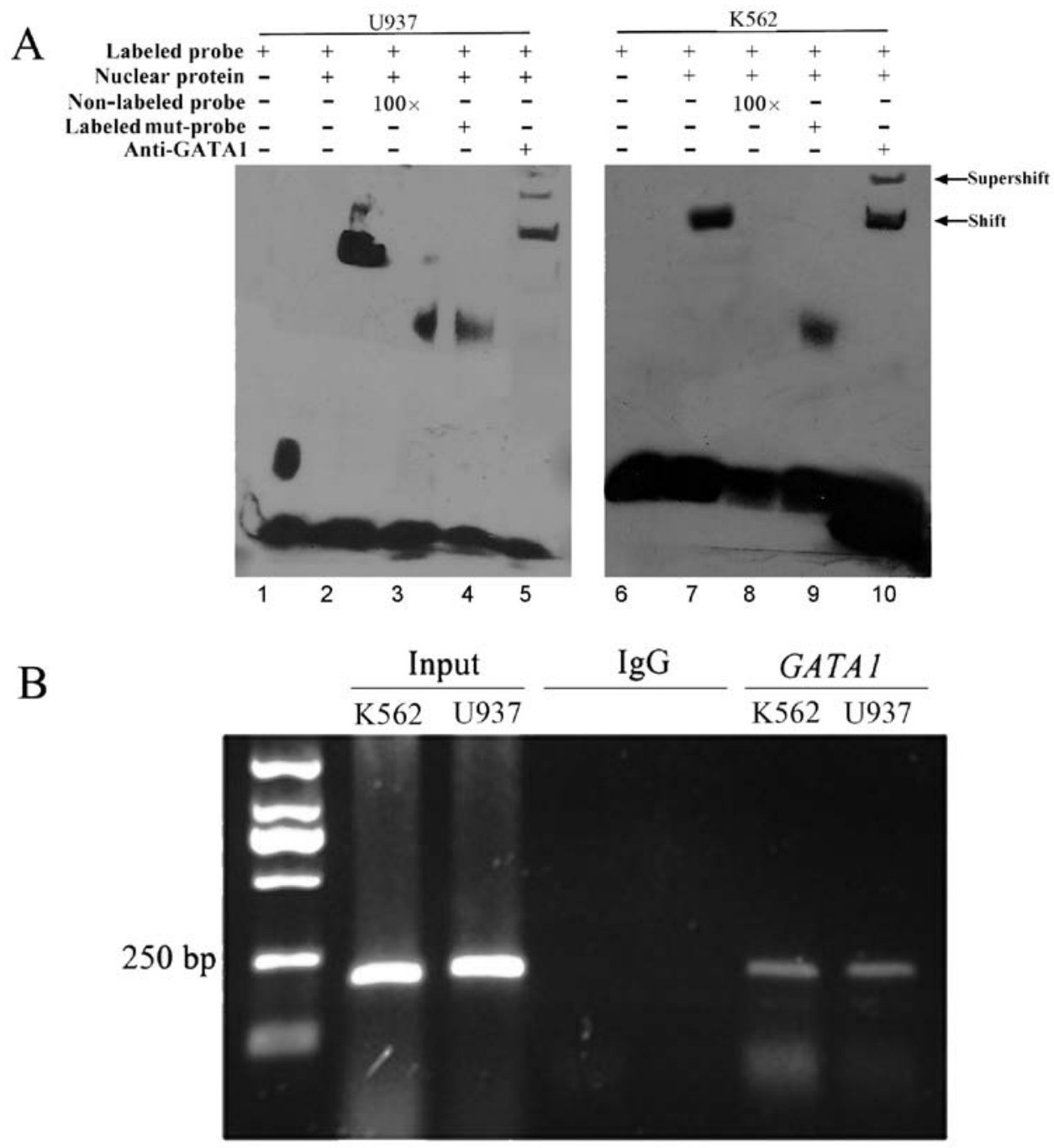

Figure 4. GATA1 binds to the DICER1 promoter. (A) DICER1 GATA1-binding sites, as detected by EMSA in vitro. Lanes 1 to 5, results from U937 cells; lanes 6 to 10, results from K562 cells; lanes 1 and 6, biotin-labeled oligonucleotides; lanes 2 and 7, probes incubated with nuclear extracts; lanes 3 and 8 , probes incubated with a 100-fold excess of the unlabeled competitor oligonucleotides; lanes 4 and 9 , probes incubated with labeled mutant oligonucleotides; and lanes 5 and 10, EMSA results in the presence of anti-GATA1 antibody. (B) DICER1 GATA1-binding sites, as detected by ChIP in vivo. The input lanes correspond to PCR products of K562 cells or U937 cells derived from chromatin prior to immunoprecipitation. The IgG lanes correspond to PCR products containing chromatin immunoprecipitated with antibodies against control IgG. The GATA1 lanes correspond to PCR products containing chromatin immunoprecipitated with antibodies against GATA1.

previous studies have demonstrated GATA1 overexpression in AML $(20,21)$. Therefore, we transfected K562 and U937 cells with GATA1-shRNA and, as shown in Fig. 6B, GATA1-shRNA downregulated the mRNA and protein levels of DICERI, as opposed to the control-shRNA. Taken together, these results lead to the conclusion that the overexpression of DICERI is induced by GATA1 in AML.

\section{Discussion}

Alterations in DICER1 expression levels in various types of cancer have been observed. In prostate cancer, precursor lesions of lung adenocarcinomas and colorectal cancer, DICERI overexpression has been observed $(5,6,22)$, while in ovarian cancer, invasive lung adenocarcinoma and breast cancer, decreased expression has been demonstrated (7-9). The dysregulation of DICER1 expression appears to be related to the aggressiveness and metastatic spread of cancer. For example, in prostate and colorectal cancer, the overexpression of DICER1 has been shown to predict poor survival $(5,11)$, whereas the downregulation of its expression has been associated with aggressiveness and the metastatic spread of breast and lung cancer $(8,9)$. These findings suggest that DICER1 plays an important role in carcinogenesis. However, the functional role of DICERI in tumors is controversial. Therefore, in this study we aimed to investigate the expression levels of DICERI in AML and the potential role of DICERI in leukemia cells. The results revealed that DICERI expression in AML patients was highly increased compared to the normal donors. The functional assays demonstrated that the silencing of DICER1 inhibited cell proliferation and promoted apoptosis in leukemia cell lines. Our results are similar to those reported by Zhou etal; their study demonstrated that the silencing of DICERI was associated with significantly enhanced G0- to G1-phase accumulation and significantly increased apoptosis in myeloma cell lines (23). Furthermore, studies on oral cancer cells have demonstrated that the overexpression of DICERI 
A
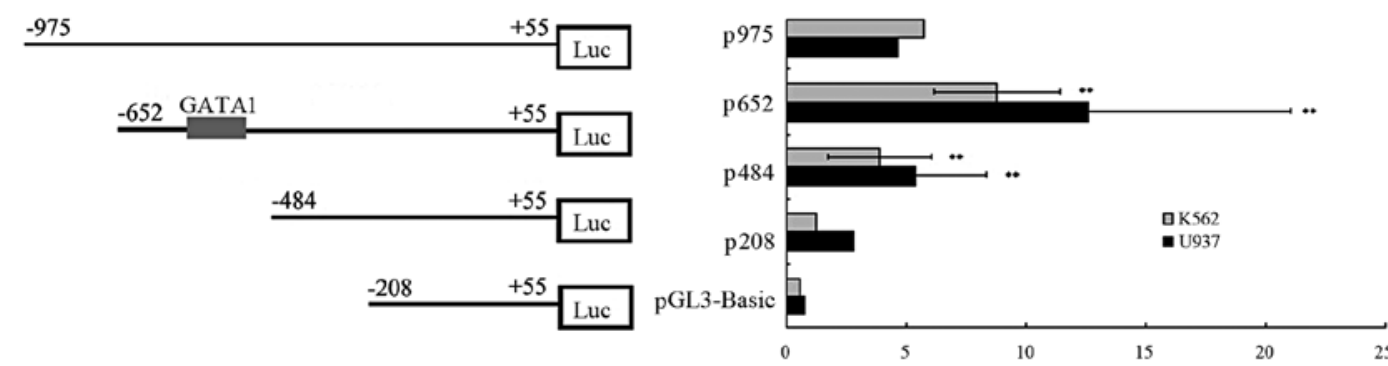

B

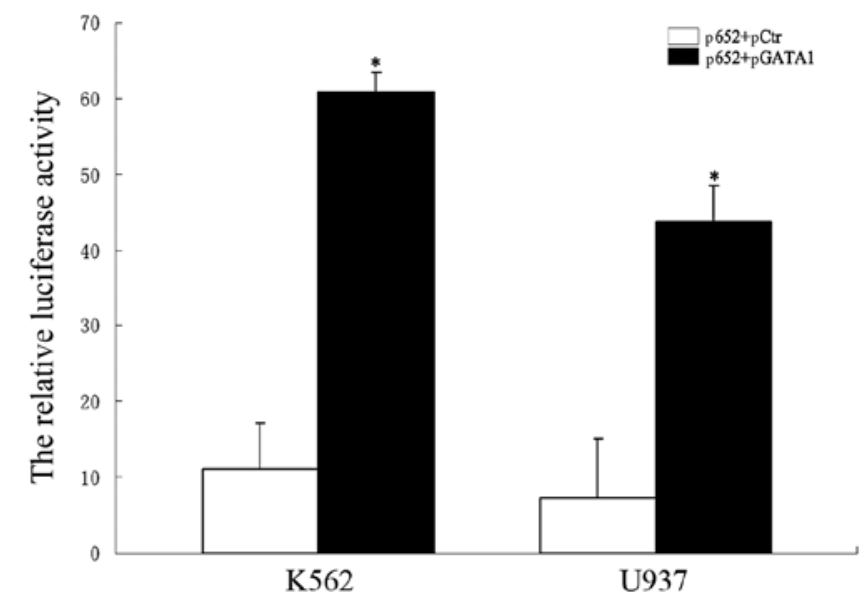

Figure 5. GATA1 transactivates the DICER1 promoter. (A) Analysis of the human DICER1 promoter activities detected by luciferase assay. Map of the recombinant vector is shown on the left panel and the relative activities of a series of deletion constructs, as determined by the luciferase assay, are shown on the right panel. The luciferase activity of p652 is increased by approximately 2 -fold as compared to that of p484. ${ }^{* *} \mathrm{P}<0.01$. (B) Relative luciferase activity in the p652 + GATA1 expression vector (pGATA1) group and the p652 + empty pcDNA3.1 (pCtr) group, at 2 days post-transfection."P<0.05.

A
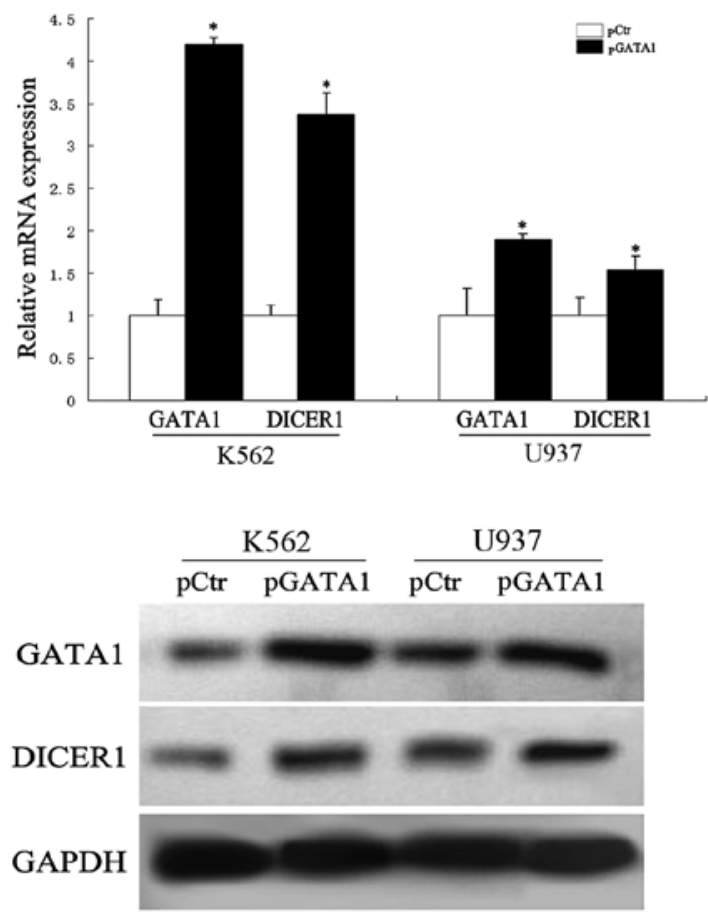

B
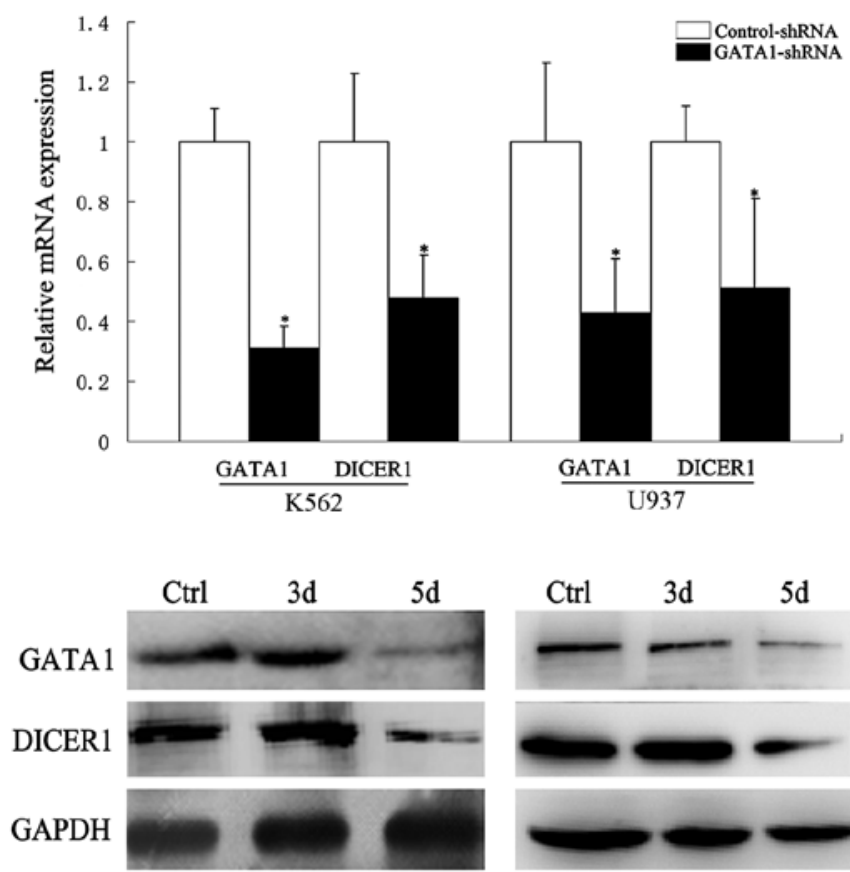

K562

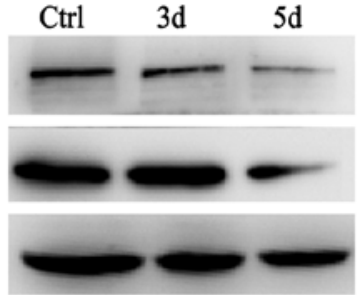

U937

Figure 6. Effects of GATA1 on DICER1 expression. (A) Overexpression of GATA1 increased DICER1 expression. When K562 and U937 cells were transfected with a GATA1 expression construct for 2 days, a significantly increased expression of DICER1 was observed. (B) Knockdown of GATA1 in the K562 and U937 leukemia cell lines. Compared to the control group, the levels of GATAl mRNA and protein were significantly decreased in both cell lines 5 days following transfection with GATA1-shRNA. DICER1 expression levels were downregulated following the inhibition of GATA1 expression by shRNA, as determined by real-time PCR and western blot analysis. ${ }^{*}<0.05$. 
increases cell proliferation (24). These data indicate that high DICERI expression may induce leukemia cell proliferation and inhibit apoptosis, mechanisms by which DICERI may promote leukemia progression. Additional studies are required in order to further elucidate the function of DICERI in tumor cells.

Although our study, as well as others have demonstrated that specific types of tumors exhibit elevated levels of DICERI, there is evidence that other types of cancer have decreased DICERI expression levels. These discrepancies may be attributed to tissue-specific differences or progression of disease. For example, in lung cancer, the upregulation of DICERI has been observed in precursor lesions and the reduced expression at the more aggressive stages (8).

Unfortunately, knowledge of the regulatory mechanisms underlying these alterations of DICERI expression levels in cancer is limited. A previous study demonstrated that the regulation of Dicer expression was mainly post-transcriptional, as cellular Dicer mRNA did not correlate well with protein expression (25). However, in breast cancer cell lines, a 72\% concordance was observed between DICERI mRNA and protein levels (9). In this study, we detected DICERI protein expression in 19 AML cases, in which high DICER1 mRNA levels were observed. The results demonstrated a $74 \%$ concordance between DICERI mRNA and protein levels, which suggested that DICER1 may be transcriptionally upregulated in AML. Levy et al identified a novel mechanism of DICER upregulation via its transcriptional targeting by the melanocytic transcription factor, microphthalmia-associated transcription factor (MITF), during melanocyte differentiation (26). In this study, we reported that DICERI expression in AML was regulated by the hematopoietic transcription factor, GATA1. GATA1 is a zinc-finger transcription factor that plays an important role in gene regulation during development and differentiation of hematopoietic cells. The tissue-specific expression of GATA1 is mainly restricted to hematopoietic (erythroid cells, megakaryocytes, eosinophils and mast cells) and Sertoli cells $(13,14)$. During normal hematopoiesis, GATA1 is not expressed in myeloid progenitor cells; however, previous studies have shown that leukocytes from AML patients express a significant amount of GATA1 (17-19). In addition, the overexpression of GATA1 has been shown to exert a negative effect on the event-free survival of AML patients $(20,21)$.

In the present study, we demonsrate that the upegulation of GATA1 affects the transcription of DICERl in AML. This finding may provide a framework for understanding the differential DICERI expression among different types of cancer. Elucidating the effects of DICERI on leukemia cell proliferation and apoptosis will aid in the understanding of its role in the development of cancer.

\section{Acknowledgements}

We thank Professor Wei-Neng Fu of the Department of Medical Genetics, China Medical University, for providing technical support and helpful suggestions.

\section{References}

1. Cerutti $\mathrm{H}$ and Casas-Mollano JA: On the origin and functions of RNA-mediated silencing: from protists to man. Curr Genet 50: 81-99, 2006.
2. Peng JJ, Yan F, Chen HR and Chen JP: Progress of studies on Dicer structure and function. Yi Chuan 30: 1550-1556, 2008 (In Chinese).

3. Lee YS, Nakahara K, Pham JW et al: Distinct roles for Drosophila Dicer-1 and Dicer-2 in the siRNA/miRNA silencing pathways. Cell 117: 69-81, 2004.

4. Filipowicz W, Jaskiewicz L, Kolb FA and Pillai RS: Posttranscriptional gene silencing by siRNAs and miRNAs. Curr Opin Struct Biol 15: 331-341, 2005.

5. Chiosea S, Jelezcova E, Chandran U, et al: Up-regulation of dicer, a component of the MicroRNA machinery, in prostate adenocarcinoma. Am J Pathol 169: 1812-1820, 2006.

6. Chiosea S, Jelezcova E, Chandran U, et al: Overexpression of Dicer in precursor lesions of lung adenocarcinoma. Cancer Res 67: 2345-2350, 2007.

7. Merritt WM, Lin YG, Han LY, et al: Dicer, Drosha, and outcomes in patients with ovarian cancer. N Engl J Med 359: 2641-2650, 2008.

8. Karube Y, Tanaka H, Osada H, et al: Reduced expression of Dicer associated with poor prognosis in lung cancer patients. Cancer Sci 96: 111-115, 2005.

9. Grelier G, Voirin N, Ay AS, et al: Prognostic value of Dicer expression in human breast cancers and association with the mesenchymal phenotype. Br J Cancer 101: 673-683, 2009.

10. Zighelboim I, Reinhart AJ, Gao F, et al: DICER1 expression and outcomes in endometrioid endometrial adenocarcinoma. Cancer 117: 1446-1453, 2011.

11. Faber C, Horst D, Hlubek F and Kirchner T: Overexpression of Dicer predicts poor survival in colorectal cancer. Eur J Cancer 47: 1414-1419, 2011.

12. Vaksman O, Hetland TE, Trope' CG, et al: Argonaute, Dicer, and Drosha are up-regulated along tumor progression in serous ovarian carcinoma. Hum Pathol 43: 2062-2069, 2012.

13. Ross J, Bottardi S, Bourgoin V, Wollenschlaeger A, Drobetsky E, et al: Differential requirement of a distal regulatory region for pre-initiation complex formation at globin gene promoters. Nucleic Acids Res 37: 5295-5308, 2009.

14. Li J, Wang E, Dutta S, Lau JS, Jiang SW, et al: Protein kinase C-mediated modulation of FIH-1 expression by the homeodomain protein CDP/Cut/Cux. Mol Cell Biol 27: 7345-7353, 2007.

15. Harigae H: GATA transcription factors and hematological diseases. Tohoku J Exp Med 210: 1-9, 2006.

16. Shimizu R and Yamamoto M: Function and gene expression regulation of GATA-1 and GATA-2 transcription. Seikagaku 79: 941-952, 2007 (In Japanese).

17. Shimamoto T, Ohyashiki JH, Ohyashiki K, et al: GATA-1, GATA-2, and stem cell leukemia gene expression in acute myeloid leukemia. Leukemia 8: 1176-1180, 1994.

18. Guerrasio A, Saglio G, Rosso C, et al: Expression of GATA-1 mRNA in human myeloid leukemic cells. Leukemia 8: 1034-1038, 1994.

19. Shimamoto T, Ohyashiki K, Ohyashiki JH, et al: The expression pattern of erythrocyte/megakaryocytes-related transcription factors GATA-1 and stem cell leukemia gene correlates with hematopoietic differentiation and is associated with outcome of acute myeloid leukemia. Blood 86: 3173-3180, 1995.

20. Ayala RM, Martínez-López J, Albízua E, et al: Clinical significance of Gata-1, Gata-2, EKLF, and c-MPL expression in acute myeloid leukemia. Am J Hematol 84: 79-86, 2009.

21. Ohyashiki K, Ohyashiki JH, Shimamoto T and Toyama K: Pattern of expression and their clinical implications of the GATA family, stem cell leukemia gene, and EVI1 in leukemia and myelodysplastic syndromes. Leuk Lymphoma 23: 431-436, 1996.

22. Papachristou DJ, Korpetinou A, Giannopoulou E, et al: Expression of the ribonucleases Drosha, Dicer, and Ago2 in colorectal carcinomas. Virchows Arch 459: 431-440, 2011.

23. Zhou Y, Chen L, Barlogie B, Stephens O, Wu X, Williams DR, et al: High-risk myeloma is associated with global elevation of miRNAs and overexpression of EIF2C2/AGO2. Proc Natl Acad Sci USA 107: 7904-7909, 2010.

24. Jakymiw A, Patel RS, Deming N, et al: Overexpression of Dicer as a result of reduced let-7 microRNA levels contributes to increased cell proliferation of oral cancer cells. Genes Chromosomes Cancer 49: 549-559, 2010.

25. Wiesen JL and Tomasi TB: Dicer is regulated by cellular stresses and interferons. Mol Immunol 46: 1222-1228, 2009.

26. Levy C, Khaled M, Robinson KC, et al: Lineage-specific transcriptional regulation of DICER by MITF in melanocytes. Cell 141: 994-1005, 2010. 